\title{
Ein Hungerstreik ist eine Protesthandlung
}

Bruno Gravier ${ }^{1}$, Hans Wolff ${ }^{2}$, Dominique Sprumont ${ }^{3}$, Bara Ricou ${ }^{4}$, Christian Kind 5 , Ariel Eytan ${ }^{6}$, Markus

Zimmermann-Acklin ${ }^{7}$, René Raggenbass ${ }^{8}$, Bernice Elger ${ }^{9}$, Helena Slama ${ }^{10}$, Marianne Wälti-Bolliger ${ }^{11}$, Philipp Weiss ${ }^{12}$, Thomas Bischoff ${ }^{13}$, Valdo Pezzoli ${ }^{14}$, Monique Gauthey ${ }^{15}$, Georg Bosshard ${ }^{16}$, Panteleimon Giannakopoulos ${ }^{17}$, Jean-Michel Gaspoz ${ }^{18}$, Alex Mauron ${ }^{19}$, Peter Suter ${ }^{20}$, Jacques de Haller ${ }^{21}$, Samia Hurst ${ }^{22}$

1-22: Zusatzangaben zu den Autoren auf Seite 1525

Vergl. zu diesem Thema auch die Zusammenstellung relevanter Richtlinien auf Seite 1527.

Unter «Pflegenden» werden sowohl Ärzte wie Pflegefachkräfte verstanden. Grundsätzlich sind sowohl Frauen wie Männer gemeint. Auf die doppelte Ausführung in weiblicher und männlicher Form wird hier zugunsten der besseren Lesbarkeit verzichtet.

Korrespondenz:

Prof. Bruno Gravier

SMPP

Site de Cery

CH-1008 Prilly

bruno.gravier@chuv.ch

\section{Einleitung}

Ein Hungerstreik ist ein menschliches Drama. Er muss als - oftmals letzter - Protestakt einer Person verstanden werden, die keine Möglichkeit sieht, sich auf andere Weise Gehör zu verschaffen.

Dieser Protestakt fordert diejenigen heraus, an bzw. gegen die er sich richtet: eine Person oder eine Gemeinschaft. Der Ausgang einer solchen Konfrontation ist notwendigerweise offen. Er wird sowohl durch die Intensität der Auseinandersetzung als auch durch die auf dem Spiel stehenden Werte beeinflusst.

Ein Hungerstreik ist auch ein kollektives und soziales Drama, das auf die öffentliche Bühne getragen wird. Dem «Publikum» wird der Wille einer urteils- und handlungsfähigen Person vorgeführt, die ihre Entscheidung frei von äusseren Zwängen getroffen hat. Die Person ist zum Äussersten bereit, bis hin zum Tod, um ihrer Haltung Ausdruck zu verleihen.

Der Hungerstreikende will nicht sterben [1]; er will vor allem, dass seine Forderung wahrgenommen wird. Er weiss, dass ein tödlicher Ausgang möglich ist, sobald die Situation zu einem unlösbaren Konflikt eskaliert. Ein Hungerstreik ist ein Skandal, stellt er doch unsere etablierten Werte auf den Kopf. In einem Land, in dem die demokratische Debatte viele andere Möglichkeiten bietet, um sich auszudrücken, ist dies schwer zu akzeptieren.

Mit den teilweise wahnwitzigen Dimensionen einer solchen Handlung sowie den damit verbundenen Emotionen geht das Risiko einher, dass unsere eigene Beurteilung entweder «verschleiert» wird oder wir sie im Gegenteil verabsolutieren und die ethischen und standesrechtlichen Werte in den Hintergrund stellen.

Innerhalb einer demokratischen Gesellschaft wird der Skandal noch dadurch verstärkt, dass er von einer inhaftierten Person ausgeht, die sich einem Gerichtsurteil widersetzt. Diese Person stellt die Legitimität einer Institution in Frage, die für eine gut funktionierende Gesellschaft unabdingbar ist und dem Bürger alle Rekursmöglichkeiten bietet. Die Legitimität der Justiz und ihrer Entscheidungen darf allerdings den menschlichen Wert dieser Handlung nicht in den Hintergrund drängen, genauso wenig wie den Respekt vor der zweifachen Verankerung - in Einzigartigkeit und gleichwertiger Zugehörigkeit -, die die menschliche Würde in ihrem Kern definiert [2].

Umso heikler ist die Position des Arztes. Es ist immer schwierig, die richtige Haltung gegenüber einem Hungerstreikenden zu finden. Unter Bedingun-

\section{Zusammenfassung}

Im August dieses Jahres entschied das Bundesgericht, dass «die Strafvollzugsbehörde eine Zwangsernährung anordnen muss, wenn dies der einzige Weg ist, irreversible Schäden oder den Tod des Gefangenen zu vermeiden». Damit rücken die Gesundheitsdienste der Gefängnisse in den Blickpunkt der Öffentlichkeit, die eine solche Anordnung gegebenenfalls auszuführen hätten. Diese Konstellation steht im Widerspruch zu den Grundwerten der medizinischen Ethik. Der Respekt der Autonomie eines urteilsfähigen Patienten, der mit einem Hungerstreik gegen seine Lage protestiert, muss ein zentrales Anliegen der Ärzte und Pflegenden bleiben. Die Unabhängigkeit der Ärzte und Pflegenden, die Respektierung der Standesregeln, die Garantie der Vertraulichkeit und das Recht auf Verweigerung medizinischer Handlungen aus Gewissensgründen gewährleisten den Schutz dieser ethischen Grundwerte; und diese Grundwerte sind für die Ausübung der medizinischen Tätigkeit im Gefängnis - wie auch in allen anderen Einrichtungen, in denen Menschen medizinisch betreut werden unabdingbar.

gen, die das für einen fruchtbaren Austausch nötige Vertrauensklima gefährden, ist für die adäquate Betreuung eines Patienten eine sorgfältige Erkundung seines tatsächlichen Willens erforderlich. Die Einschätzung seiner Urteilsfähigkeit, die durch das Fasten beeinträchtigt sein kann, muss äusserst sorgfältig erfolgen [3]. Erschwerend ist auch das Spannungsfeld zwischen medizinischer Verpflichtung gegenüber dem Patienten und Loyalität gegenüber dem Auftrags- oder Arbeitgeber, in dem sich Ärzte und Pflegende bewegen. Die Probleme, die sich daraus ergeben, können unter Umständen unlösbar werden.

Kann man - oder muss man - eine inhaftierte Person medizinisch anders als einen freien Bürger behandeln? Die Antwort auf diese zentrale Frage ist ein deutliches Nein. Einer der Grundsätze, die für die Aus- 
übung der ärztlichen Tätigkeit im Gefängnis gelten, besagt, dass eine inhaftierte Person Anrecht auf dieselbe Qualität und Kontinuität der ärztlichen und pflegerischen Versorgung hat, wie sie auch der Allgemeinbevölkerung des betreffenden Landes zusteht. Diese Forderung ist in den internationalen und nationalen Empfehlungen $[4,5]^{*}$ aufgeführt und gilt auch für alle Fragen, welche die Autonomie des Patienten betreffen. Jede urteilsfähige Person darf freiwillig ihr Leben auf das Spiel setzen, selbst wenn dies zum Ziel hat, die Justiz oder die Politik unter Druck zu setzen.

Die Wahrung der Verhältnismässigkeit ist im Gefängnis von besonderer Bedeutung: Auch wenn einige Rechte eingeschränkt sind, wie z.B. die Bewegungsfreiheit oder die freie Wahl eines Arztes, sind andere Rechte unbedingt zu gewährleisten, insbesondere die Einwilligung in die für die Gesundheit der inhaftierten Person nötigen Massnahmen [6].

Medizinische Zwangsmassnahmen, die einen urteilsfähigen Patienten betreffen, können nur dann zugelassen werden, wenn Dritte vor einer Gefahr geschützt werden müssen und sofern eine rechtliche Grundlage vorhanden ist. Dies betrifft z. B., unter sehr restriktiven Bedingungen, die Prävention von Gewalthandlungen psychisch abnormer Personen. Hierbei handelt es sich aber um eine Ausnahme. In diesem Zusammenhang muss betont werden, dass es das Epidemigesetz nicht erlaubt, eine ansteckende Person zum Schutz der Bevölkerung gegen ihren Willen zu behandeln. Eine solche Person darf lediglich isoliert werden. Es gibt sogar einige Länder, die die Zwangsbehandlung formell verbieten, wie z.B. Deutschland (Absatz 28 des Infektionsschutzgesetzes vom 20. Juli 2000) [7].

Es wäre unvorstellbar, einen Bürger zwangsweise zu ernähren, der zu Hause oder öffentlich in einen Hungerstreik getreten ist, um sich gegen eine Bestimmung zu wehren, die seine Person oder sein Hab und Gut betrifft, oder um einem öffentlichen Entscheid zu widersprechen, den er als nicht legitim empfindet.

Die Zwangsernährung ist keine einfache Lösung für ein komplexes Problem, sondern ein mit Gewalt einhergehender Eingriff mit erheblichen Risiken für die Gesundheit der zu ernährenden Person. Wird eine Zwangsernährung bei einer Person vorgenommen, die bei Bewusstsein oder die bewusstlos ist? Muss diese Person sediert werden, damit die Zwangsernährung vorgenommen werden kann? Wer fesselt die Person: ein Pflegender oder ein Vertreter der Staatsgewalt? Wie können Verletzungen vermieden werden, wenn sich die Person wehrt? Es ist offensichtlich, wie schwerwiegend solche Handlungen gegen Ethik und Respekt verstossen.

Die Pressemitteilung des Bundesgerichts vom 26. August $2010^{\star *}$, die als Anordnung für eine Zwangsernährung gegen den Willen eines urteilsfähigen Patienten verstanden werden kann, ist verwirrend und erfordert eine erneute und vertiefte Auseinandersetzung mit den ethischen Regelungen.

\section{Rückblick}

Nachdem ein Häftling zu fünf Jahren und acht Monaten Freiheitsstrafe verurteilt worden war, begann er im März 2010 einen Hungerstreik. Nach etwa hundert nicht aufeinanderfolgenden - Tagen, wurde er ins Universitätsspital Genf und später ins Inselspital Bern eingewiesen.

Diese Universitätskliniken verfügen über Einheiten für Häftlinge, die stationär ins Spital eingewiesen werden müssen. In Übereinstimmung mit den Regeln der medizinischen Ethik und den Richtlinien der Schweizerischen Akademie der Medizinischen Wissenschaften (SAMW) haben sich beide Krankenhäuser geweigert, der Forderung der betroffenen Behörden nachzukommen, den Inhaftierten zwangsweise zu ernähren. Die Strafe wurde ein erstes Mal unterbrochen und dann bei ihm zu Hause vollzogen.

Am 26. August hat das Bundesgericht (BG) das Gesuch dieses Gefangenen um Unterbrechung der Strafe abgewiesen und regte eine Zwangsernährung an, «um sein Leben zu erhalten». Das Gericht notierte, dass nur die Kantone Neuenburg, Zürich und Bern über ein Gesetz verfügen, das eine Zwangsernährung ausdrücklich erlaubt. Stimmen wurden laut, dass das Parlament dieses Dossier in die Hand nehmen sollte.

Das Bundesgericht präzisiert nicht, wer die Zwangsernährung vornehmen soll. Es besteht aber kein Zweifel daran, dass die mit der Betreuung eines Inhaftierten im Hungerstreik beauftragten Ärzte früher oder später mit einer solchen Frage konfrontiert sein werden.

\section{Die Notwendigkeit der Unabhängigkeit medizinischer Fachpersonen, die mit der Betreuung von inhaftierten Personen beauftragt sind}

Es ist begreiflich, dass die Behörden die Gesundheit und das Leben eines Hungerstreikenden, für den sie als Strafvollzugsbehörde verantwortlich sind, wohlwollend schützen wollen. Heisst das aber, dass die Regeln der ärztlichen Kunst und die Pflichten gegenüber seinem Patienten durch die Staatsräson oder eine richterliche Anordnung ausser Kraft gesetzt werden? Auch hier ist die Antwort klar nein. «Der Arzt ist tief mit der berufseigenen Tradition verbunden und wird von ihr gestützt; diese verlangt, dass er den authentischen Willen eines Patienten voll und ganz respektiert» [8], sofern der Patient dadurch niemanden gefährdet und er seinen Willen bei klarem Bewusstsein geäussert hat.

Die Schweiz hat, über die ärztliche Tradition hinaus, die Texte des Europarates ratifiziert, die einerseits die Gewährleistung der beruflichen Unabhängigkeit der im Gefängnis tätigen Ärzte und Pflegenden empfehlen und andererseits bestätigen, dass «die gesundheitlichen Bedürfnisse des Inhaftierten für den Arzt immer im Vordergrund stehen sollten» und «die klinischen Entscheide und alle anderen Erwägungen, die die Gesundheit der inhaftierten Personen betreffen, 
ausschliesslich auf medizinischen Kriterien beruhen sollten» [9].

Die Unabhängigkeit der Ärzte und Pflegenden im Gefängnis gegenüber den Gefängnis-, Justiz- und Polizeibehörden ist somit grundlegend [6]. Sie erlaubt es, von Interessenskonflikten weitgehend verschont zu bleiben, die in Institutionen mit hoher Verfügungsgewalt zu beobachten sind.

Die Bedeutung der Unabhängigkeit des Medizinalpersonals wird besonders deutlich in der vorliegenden Situation, in der die medizinische Fachperson zwischen zwei Alternativen wählen muss: entweder die freie Wahl eines urteilsfähigen Patienten zu respektieren, der seinen Willen klar geäussert und in einer regelmässig in Einzelgesprächen bestätigten Patientenverfügung festgehalten hat, oder der auf einem Gerichtsentscheid beruhenden Anordnung einer Gefängnisbehörde zu folgen und den Patienten zwangsweise zu ernähren.

$\mathrm{Zu}$ unterstreichen ist dabei die Notwendigkeit, dass solche Einzelgespräche zwischen dem Arzt und seinem Patienten in absoluter Vertraulichkeit stattfinden können; und dies unabhängig von den öffentlichen Aussagen des Hungerstreikenden. Nur auf der Basis eines solchen Vertrauensverhältnisses ist es für den Behandelnden möglich, die verschiedenen oben erwähnten Parameter verlässlich zu bewerten. Zudem erlaubt es ihm zu beurteilen, wie die Willensäusserungen des Hungerstreikenden sich entwickeln, was insofern von Bedeutung ist, als Patientenverfügungen jederzeit geändert werden können.

Ohne Unabhängigkeit und die Möglichkeit, die Interessen der Patienten ins Zentrum ihrer Überlegungen zu stellen, werden die medizinischen Betreuer zu Vertretern der Staatsgewalt - eine Rolle, die nicht die ihre sein kann.

Kein Gerichtsentscheid kann die zentrale Dimension der Funktion der Ärzte und Pflegenden aufheben; diese beruht vor allem auf der Arzt-Patienten-Beziehung, die um jeden Preis respektiert werden muss.

\section{Die Zwangsernährung widerspricht dem ärztlichen Berufsethos}

Die Pflicht von Ärzten und Pflegenden ist es, die Einstellung ihrer Patienten zur Behandlung zu respektieren, auch wenn sie deren Entscheidungen nicht teilen. Wie oben erwähnt, darf die Behandlung einer Person nicht zwangsmässig erfolgen. Ausnahmefälle sind gegeben, wenn eine Person urteilsunfähig ist oder die Betreuung zum Ziel hat, eine Gefahr zu vermeiden, z.B. Gewalt gegenüber Dritten. Die Behandlung muss auch das Kriterium der Verhältnismässigkeit respektieren.

Die Zwangsernährung eines urteilsfähigen Hungerstreikenden erfüllt diese Bedingungen nicht. Sie widerspricht dem Recht des Streikenden auf Selbstbestimmung und körperliche Integrität und somit dem ärztlichen Berufsethos. Wenn eine Ernährung unter Zwang und mit den erwähnten Risiken erfolgt, erhält die Frage nach dem unmenschlichen Charak- ter einer solchen Handlung sowie der Gewalt, die dem Patienten angetan wird, grosses Gewicht.

Aus diesem Grund haben die SAMW, die FMH, der Europarat, die World Medical Association (WMA) zur Frage des Hungerstreiks schon mehrere Stellungnahmen verfasst. In der Erklärung von Malta hat die WMA unmissverständlich bestätigt: «Die Zwangsernährung trotz freiwilliger und erklärter Verweigerung ist nicht vertretbar (...) Die Zwangsernährung ist nie akzeptabel. Selbst mit karitativer Zielsetzung ist die Ernährung unter Drohungen oder Zwang und mit Hilfe von Gewalt oder physischer Ruhigstellung eine Form von unmenschlicher und erniedrigender Behandlung» [10].

Der Europäische Gerichtshof für Menschenrechte hat in zwei Urteilen bestätigt, dass die Zwangsernährung als Folter betrachtet werden kann, wenn dem Inhaftierten Fesseln angelegt werden oder wenn ihm zwangsmässig eine Ernährungssonde eingelegt wird $[11,12]$. In einem anderen Urteil hat dasselbe Gericht entschieden, dass der Tod eines Gefangenen nach einem Hungerstreik nicht gegen die Menschenrechte verstösst, weil er während seiner Gefangenschaft Zugang zur gleichen Behandlung wie in der Freiheit hatte [13].

\section{Der Arzt, der Hungerstreik und die Verweigerung aus Gewissensgründen} Die SAMW hält für den Fall eines Hungerstreiks unter Haftbedingungen fest: «(...) nachdem die volle Urteilsfähigkeit der betreffenden Person von einem ausserhalb der Anstalt tätigen Arzt bestätigt wurde, muss der Entscheid zum Hungerstreik, auch im Falle eines beträchtlichen Gesundheitsrisikos, medizinisch respektiert werden.» Und weiter: «(...) fällt die Person im Hungerstreik in ein Koma, geht der Arzt nach seinem Gewissen und seiner Berufsethik vor, es sei denn, die betreffende Person habe ausdrückliche Anordnungen für den Fall eines Bewusstseinsverlustes hinterlegt, auch wenn diese den Tod zur Folge haben können» [5].

Eine künstliche Ernährung wäre also möglich, aber nur wenn der Patient nicht urteilsfähig ist und keine klaren Anweisungen hinterlassen hat. In diesem Sinne unterscheiden sich die Hungerstreikenden nicht von anderen Patienten, die lebenserhaltende Massnahmen verweigern.

Ausserdem können diese Personen - wie jeder freie Bürger - ermutigt werden, eine Patientenverfügung zu erstellen, um ihren Willen für den Fall festzuhalten, dass sie ihre Urteilsfähigkeit verlieren. Die Folgen des Hungerstreiks sind voraussehbar. Bei länger dauerndem Hungerstreik ist es wichtig, alle möglichen Vorsichtsmassnahmen zu ergreifen, um sicherzustellen, dass die Patientenverfügung den tatsächlichen Willen eines urteilsfähigen Patienten widerspiegelt. Dieses Vorgehen muss der erhöhten Komplexität der Umstände im Gefängnis und den Beweggründen des Streikenden Rechnung tragen. Bei der Erstellung einer Patientenverfügung soll der Streikende die Möglich- 
keit haben, seine Behandlungswünsche oder die Verweigerung einer Behandlung einem Arzt und/oder seiner Vertrauensperson, unter strenger Wahrung der Vertraulichkeit, mitzuteilen. Auf diese Weise kann seine Autonomie auch im Falle des Bewusstseinsverlusts respektiert werden.

Eines steht fest: Eine hungerstreikende Person will nicht sterben [1]. Aber sie will «lieber sterben, als ...». Der Arzt muss sich vergewissern, dass die Entscheidung ohne äusseren Druck getroffen wurde, dass sie also frei handelt. Zudem muss er die Urteilsfähigkeit seines Patienten sorgfältig evaluieren. Es gehört auch zu den Aufgaben klinischer Ethikkommissionen, den behandelnden Arzt in seinen Entscheidungen zu unterstützen. Das Einholen einer Zweitmeinung kann ebenfalls hilfreich sein. Es handelt sich in solchen Fällen um schwerwiegende Entscheidungen, die kein Arzt leichtfertig trifft. Die ethischen Empfehlungen sollen dem Kliniker eine Hilfe bieten; können aber eine sorgfältige klinische Abwägung ebenso wenig ersetzen wie eine vertiefte Reflexion und die unausweichliche Konfrontation mit den eigenen Grundwerten.

Wenn die medizinische Beurteilung ergibt, dass der Hungerstreikende urteilsfähig ist und dass er seine Entscheidung frei getroffen hat, muss seine Autonomie respektiert werden, auch wenn die betreuenden Medizinalpersonen seiner Wahl selbst nicht zustimmen. Das Respektieren des unzweideutig geäusserten Willens ist ein Gebot des ärztlichen und pflegerischen Berufsethos. Die Ärzte und Pflegenden verpflichten sich, ihre Kenntnisse und Fertigkeiten für das Wohl der kranken Menschen einzusetzen. Dies bedeutet, dass die Interessen ihrer Patienten im Vordergrund stehen. Dieses Prinzip der medizinischen Ethik ist grundlegend, weil medizinische Methoden und Instrumente, zur Erreichung anderer - oft nicht vertretbarer - Ziele zweckentfremdet werden können. Nur wenn die Arzt-Patienten-Beziehung im Zentrum behalten wird, kann ein verantwortungsvoller Umgang mit den wissenschaftlichen und technischen Möglichkeiten der Medizin garantiert werden. Wenn eine Behörde die medizinischen Betreuungspersonen zwingen will, gegen dieses Prinzip zu verstossen, müssen diese Stellung nehmen und ihr Recht auf Verweigerung aus Gewissensgründen geltend machen. Spannungsfelder zwischen den rechtlichen Entscheiden und dem ärztlichen Berufsethos sind nicht selten und betreffen auf internationaler Ebene vor allem die ärztliche Beteiligung an Folter [14] und Todesstrafe [15] sowie an der Inhaftierung von politischen Gefangenen in psychiatrischen Einheiten [16]. In der Schweiz ist das Recht auf Verweigerung ärztlich-pflegerischer Handlungen aus Gewissensgründen geschützt [17]. Das ärztliche Berufsethos wird in Rechtsstaaten generell anerkannt und respektiert. In den USA, wo die ärztliche Beteiligung an der Todesstrafe erhebliche Spannungen verursacht, wurde bisher kein Arzt verurteilt, weil er die Kooperation verweigerte, und dies sogar in Staaten, in denen das Gesetz die Teilnahme der Ärzte ausdrücklich vorsieht.
Die Weigerung der Ärzte und Pflegenden, zu einem Instrument der Staatsmacht zu werden, beruht nicht auf einer Beurteilung der Angemessenheit des zur Debatte stehenden Urteils gegenüber dem Hungerstreikenden. Ausschlaggebend für diese Weigerung ist allein die Tatsache, als medizinische Fachperson durch ihre Mitwirkung Teil der Staatsmacht zu werden. Die Weigerung basiert auf der Überzeugung, dass die Medizin nicht für Zwecke missbraucht werden darf, die gegen die Interessen der Patienten verstossen, und auf der Forderung, Medizin und Justiz zu trennen.

\section{Polizeiliche Generalklausel und Patientenverfügung}

Bereits im Jahre 2000 wurde vom Bundesgericht die polizeiliche Generalklausel angewandt, damals, um die Zwangsbehandlung eines psychiatrischen Patienten zu begründen [18]. Bevor wir dazu Stellung nehmen, sollte die polizeiliche Generalklausel noch präzisiert werden. Es handelt sich um einen Ausnahmezustand, in dem der staatliche Eingriff ohne gesetzliche Grundlage erlaubt ist, um ernsten, unmittelbaren und nicht anders abwendbaren Gefahren zu begegnen (Artikel 36 der Bundesverfassung). Weil dabei das Legalitätsprinzip in Frage gestellt wird, kann diese Klausel nur bei ausserordentlichen Umständen, die die öffentliche Ordnung gefährden, in Erwägung gezogen werden $[19,20]$. Diese Klausel wird z. B. berücksichtigt, um die Gefahr eines Terroranschlags oder öffentlicher Gewalt abzuwenden oder um ausserordentliche Massnahmen zu ergreifen, z. B. zur Bekämpfung einer Epidemie (Artikel 10, Epidemiegesetz).

Im Urteil des Bundesgerichts betreffend die Behandlung eines psychiatrischen Patienten wurden restriktive Bedingungen definiert. Erstens stellte der Patient aufgrund seiner Aggressivität (die auf seine Krankheit zurückzuführen war) ein hohes Risiko für die Pflegenden und die anderen Patienten dar; zweitens war er urteilsunfähig und hatte keine Patientenverfügung erstellt. Dazu muss ergänzt werden, dass die polizeiliche Generalklausel keinen unverhältnismässigen und dem unantastbaren Kern der Grundfreiheiten widersprechenden Verstoss zulässt. Bezüglich des Hungerstreiks ist die erste Bedingung - die Gefahr für Dritte - nicht erfüllt; die Art und Weise einer Zwangsernährung wurde vom Europäischen Gerichtshof für Menschenrechte der Folter gleichgestellt.

Schliesslich ist zu betonen, wie beunruhigend es ist, dass das Bundesgericht wenig Wert auf die Patientenverfügung zu legen scheint. Die Willenserklärungen des Patienten sind für die Behandelnden verbindlich. Wenn das Urteil des Bundesgerichts einer Grundregel widerspricht, die seit Jahren im Medizinalrecht verwendet wird, dann stellt es die in den letzten 30 Jahren auf kantonaler, nationaler und internationaler Ebene entwickelten Patientenrechte in Frage. Das kann nicht ohne Folgen bleiben, da die Ärzte und Spitäler heutzutage mit vielen Anfragen von Patien- 
ten konfrontiert werden, die sich Sorgen machen, ob ihrem Willen tatsächlich entsprochen wird.

\section{Schlussfolgerungen}

Die SAMW, die FMH, der Europarat und die World Medical Association haben einstimmig zur Frage des Hungerstreiks Stellung bezogen.

Mit dem Thema Zwangsernährung darf nicht leichtfertig umgegangen werden. Wenn dabei Gewalt angewendet wird, stellt die Zwangsernährung eine unmenschliche Handlung dar. In allen Fällen verstösst die Zwangsernährung einer sich im Hungerstreik befindenden, urteilsfähigen Person, die ihre Entscheidung frei getroffen hat, gegen das ärztliche Berufsethos. Hier muss man sich auch fragen, ob ein Arzt, der sich trotz allem der Anordnung des Bundesgerichts unterwirft, nicht nur die standesrechtlichen Normen verletzt, sondern auch den Artikel 40 des Medizinalberufegesetzes, das vom Arzt verlangt, die Rechte der Patienten zu wahren.

Diese Überlegungen sollen die Wichtigkeit der aktuellen Debatte in der schweizerischen Gesellschaft betonen, die mit einer Handlung konfrontiert ist, deren Tragweite bei weitem über die Rubrik «Vermischte Meldungen» hinausgeht. Gesellschaftsdebatte, Konfrontation der Werte: Die Reaktion der öffentlichen Institutionen auf eine solche Herausforderung lässt niemanden gleichgültig. Was immer die Beweggründe eines Hungerstreikenden sind und wie legitim auch die Haltung der Gesetzesvertreter sein mag: Der Betreuende befindet sich in einer Spagatposition. Er muss im Dienst seines Patienten handeln, hat aber auch eine

1 Professeur, Médecin chef de Service, Service de médecine et psychiatrie pénitentiaires/ Département de Psychiatrie, Centre Hospitalier Universitaire Vaudois et Université de Lausanne, Président de la Conférence des médecins pénitentiaires suisses.

2 Médecin responsable de l'Unité de médecine pénitentiaire, Hôpitaux Universitaires de Genève; Vice-président de la Conférence des médecins pénitentiaires suisses.

3 Professeur en droit, Directeur-adjoint de I'Institut de droit de la santé, Université de Neuchâtel.

4 Médecin adjointe agrégée, Soins intensifs, Hôpitaux Universitaires de Genève, membre du comité de la Société Suisse d'Ethique Biomédicale, membre de la Commission centrale d'éthique de I'ASSM.

5 Professor, Chefarzt Ostschweizer Kinderspital St. Gallen und Präsident der Zentralen Ethikkommission der SAMW.

6 Médecin responsable de I'Unité de psychiatrie pénitentiaire, Hôpitaux Universitaires de Genève.

7 Dr. theol., Lehr- und Forschungsrat, Departement für Moraltheologie und Ethik, Universität Fribourg, Mitglied im Vorstand der Schweizerischen Gesellschaft für Biomedizinische Ethik, Vizepräsident der Zentralen Ethikkommission der SAMW.

8 Médecin-chef du Service de médecine pénitentiaire valaisan.

9 Professeure, Centre universitaire romand de médecine légale, Université de Genève, membre du comité de la Conférence des médecins pénitentiaires suisses.

10 Dr. med. EMBA, Secrétaire de la Conférence des médecins pénitentiaires suisses.

$11 \mathrm{MSCN}$, Professeure HES.

12 Dr. med., Mitglied der ZEK und EKBB.

13 Professeur, Institut universitaire de médecine générale, Lausanne, membre de la Commission centrale d'éthique de l'ASSM.

14 Pédiatre, membre du comité de la Société Suisse d’Ethique Biomédicale, membre de la Commission centrale d'éthique de l'ASSM.

15 Médecin spécialiste pédopsychiatrie, membre du comité central FMH.

16 Facharzt FMH für Allgemeinmedizin spez. Geriatrie, Privatdozent für Klinische Ethik, Mitglied der Zentralen Ethikkommission der SAMW.

17 Professeur, Médecin-chef du Département de Psychiatrie, Hôpitaux Universitaires de Genève.

18 Professeur, Médecin-chef du Département de Médecine Communautaire, de Premier Recours et des Urgences, Hôpitaux Universitaires de Genève.

19 Dr rer. nat., Professeur ordinaire de bioéthique, Faculté de médecine, Université de Genève.

20 Professeur, Président de l'Académie Suisse des Sciences Médicales.

21 Président de la FMH.

22 Professeure, Institut d'éthique biomédicale, Faculté de médecine de Genève, présidente de la Société Suisse d'Ethique Biomédicale, membre de la Commission centrale d'éthique de l'ASSM.
Loyalitätspflicht gegenüber dem Gesetzgeber. Nur die Orientierung an ethischen und standesrechtlichen Werten kann ihm helfen, diesen unlösbaren Konflikt zu lösen. Es ist unabdingbar, den Raum der Entscheidungsfindung und -hilfe von gerichtlichen Verfügungen möglichst frei zu halten. Es geht um die Grundfesten der Medizin.

\section{Literatur}

1 Reyes H. Medical and Ethical Aspects of Hunger Strikes in Custody and the Issue of Torture. In: Oehmichen M (ed.). Maltreatment and Torture. Lübeck: Verlag Schmidt-Römhild; 1998.

2 Delmas-Marty M. L'interdit et le respect: comment définir le crime contre l'humanité. In: Colin M. Gravier B, Elchardus JM. Le Crime contre l'Humanité. Ramonville St Agne: Erès; 1996.

3 Fessler DM. The implications of starvation induced psychological changes for the ethical treatment of hunger strikers. J Med Ethics. 2003; Aug;29(4):243-7.

4 Guilbert P, Sebo P, Elger B, Bertrand D. Jeûne de protestation. in Ummel M., Bertrand D (ed.). Médecin et Santé en Prison. Genève: Médecine \& Hygiène; 2007.

5 Académie Suisse des Sciences Médicales. Directives médico-éthiques pour l'exercice de la médecine auprès de personnes détenues. 2002.

6 Sprumont D et al. Pratique médicale en milieu de détention: Effectivité des directives de l'Académie suisse des sciences médicales sur «L'exercice de la médecine auprès de personnes détenues», Rapport à la FMH (2009) (www.fmh.ch/files/pdf3/Rapport.pdf).

7 Hänni C, Harbarth S, Sprumont D. La résistance aux antibiotiques: quels enjeux juridiques? Rapport IDS $\mathrm{N}^{\circ}$ 9; 2006. p. 66ss et auteurs cités.

8 Bernheim J. Ethique en médecine pénitentiaire, Méd et Hygiène. 1991;49:2494-501.

9 Recommandation $\mathrm{N}^{\circ} \mathrm{R}(98)$ 7, 1998 concernant les aspects éthiques et organisationnels des soins de santé en milieu pénitentiaire (Comité des Ministres du Conseil de l'Europe).

10 Association Médicale Mondiale. Déclaration sur les grévistes de la faim (Déclaration de Malte) 2007: Disponible: www.wma.net/fr/30publications/ 10policies/h31/index.html.

11 CEDH. Nevmerthistsky vs. Ukraine, n 54825/00; 2005.

12 CEDH. Ciorap vs. Moldova, $n^{\circ}$ 12066/02; 2007.

13 CEDH. Horoz vs. Turkey, n 1639/03; 2009.

14 Miles SH. Medical ethics and torture: revising the Declaration of Tokyo. Lancet. 2009;373:344-8.

15 Groner JI. Lethal injection: a stain on the face of medicine. BMJ. 2002;325;1026-8.

16 Ramsay S. Human-rights group calls on China to improve psychiatric standards. Lancet. 2002;360:627.

17 Brunner N, Sprumont D. Droits des professionnels de la santé: l'objection de conscience. Cahiers de l'IDS Nº ; 2001 (www2.unine.ch/webdav/site/ids/ shared/documents/professionels_sante/objectionconscience.pdf).

18 Arrêt du 23 mai 2000 de la $1^{\text {re }}$ Chambre de droit public du Tribunal fédéral, ATF 126 I 112.

19 Moor P. Droit administratif. vol. I, Les fondements généraux. Berne; 1994. p. 337ss.

20 Häfelin U, Haller W. Schweizerisches Bundesstaatsrecht. 4. Auflage. Zürich; 1998. p. 390. 